\title{
Social Entrepreneurship im etablierten Wohlfahrtsstaat. Aktuelle empirische Befunde zu neuen und alten Akteuren auf dem Wohlfahrtsmarkt.
}

\author{
Rolf G. Heinze/Anna-Lena Schönauer/Katrin Schneiders/Stephan Grohs / \\ Claudia Ruddat
}

\section{Einleitung}

Im internationalen Vergleich hat sich die wissenschaftliche Diskussion um die gesellschaftliche Relevanz des Phänomens „Social Entrepreneurship“ (SE) in Deutschland relativ spät entwickelt. In Asien wurde die Debatte insbesondere durch die von Muhammad Yunus 1983 gegründete Grameen Bank angestoßen und spätestens seit der Auszeichnung Yunus' mit dem Friedensnobelpreis wird $\mathrm{SE}$ in vielen Nationen zunehmend als Chance wahrgenommen, soziale Missstände effektiv und nachhaltig zu bekämpfen. Insbesondere in den angelsächsischen Staaten war ein regelrechter Hype zu beobachten (vgl. z. B. Bornstein2007; Nicholls 2006; kritisch Edwards 2010).

Angeregt und finanziell gefördert wurde die deutsche Debatte insbesondere durch Stiftungen (z. B. Stiftung Mercator, Vodafone-Stiftung, Siemens-Stiftung, Schwab) und Verbände (Ashoka). Neben Medien und Politik hat das Phänomen mittlerweile auch die wissenschaftlichen Diskurse erreicht(vgl. die Beiträge in Hackenberg/Empter 2011 und Jähnke et al. 2011). Die verzögerte Aufnahme der Diskurse in Deutschland ist u. a. auf unterschiedliche wohlfahrtsstaatliche Settings zurückzuführen; während Social Entrepreneurship in Entwicklungs- und Schwellenländern und in Grenzen auch in angelsächsischen Industriestaaten auf Systeme defekter, defizitärer oder weitgehend privater Erbringung sozialer Dienstleistungen trifft, besteht in Deutschland eine gewachsene Tradition staatlich oder durch Wohlfahrtsorganisationen erbrachter sozialer Dienste (vgl. Bode/Evers 2004 sowie die Beiträge in Evers et al. 2011). Daher stellt sich nicht nur aus wissenschaftlicher Perspektive die Frage, welche Rolle dieser ,neuen“ Form sozialer Aktivitäten in einem etablierten Wohlfahrtsstaat zukommen kann. Grundsätzlich ist davon auszugehen, dass stark ausgeprägte wohlfahrtsverbandliche Struktu- 
ren, wie sie den deutschen Wohlfahrtsstaat weiterhin prägen, Aktivitäten ,,neuer" Akteure erschweren.

Allerdings lässt sich in den letzten Jahren eine gewisse Durchlässigkeit dieser korporatistischen Strukturen beobachten. Ausgelöst durch veränderte institutionelle Rahmenbedingungen haben sich die Trägerstrukturen des sozialen Dienstleistungssektors erheblich gewandelt. Erkennbar sind sowohl trägerinterne Umstrukturierungen als auch Verschiebungen zwischen den einzelnen Trägertypen sowie das Entstehen neuer Trägerformen. Während sich die öffentliche Hand aus einigen Handlungsfeldern sukzessive zurückgezogen hat (bspw. der stationären Altenpflege), übernimmt sie in anderen Feldern (bspw. der Kinder- und Jugendhilfe) neue Aufgaben (vgl. Grohs 2010; Schneiders 2010). Aufgrund unterschiedlicher Entwicklungen in den genannten sozialpolitischen Handlungsfeldern wird davon ausgegangen, dass der Bereich der Altenhilfe und -pflege einen größeren Spielraum für SEs bietet als der Bereich der Kinder- und Jugendhilfe, der sich durch eine starke Persistenz der etablierten Strukturen in den öffentlich finanzierten Bereichen auszeichnet.'Mit SEs tritt neben die „etablierten“ Träger ein (vermeintlich) neuer Trägertyp, der für sich in Anspruch nimmt, durch Verknüpfungen von sozialem Engagement und unternehmerischem Handeln die Effektivität sozialer Dienstleistungen zu verbessern. An diese sogenannten „Social Entrepreneurs" wird von einem Teil der Wissenschaft und Öffentlichkeit die Hoffnung geknüpft, durch die Integration unternehmerischer Prinzipien das Finanzierungs- und Innovationsproblem sozialpolitischer Leistungen zu lösen (vgl. zusammenfassend Heinze et al. 2011). Ein wichtiges Kennzeichen von SE ist ihre Innovationsfähigkeit, die sich darin äußert, dass SEs soziale Probleme durch ,neue“ Dienstleistungen (Produktinnovationen) oder innerhalb neuer Organisationsformen (Prozessinnovationen) besser lösen als etablierte Akteure bzw. Strukturen. Die Dritte-Sektor-Theorie und die Innovationstheorie im öffentlichen Sektor (vgl. u. a. Borins 2001; Mulgan/Albury 2003; Zimmer 2007)unterstreichen jedoch, dass Innovationen im sozialen Sektor nicht nur durch neue Akteure - Social Entrepreneurs - hervorgerufen werden. Nicht zuletzt durch den zunehmenden Wettbewerb mit anderen Anbietern, aber auch aufgrund ihres eigenen professionellen Verständnisses sind die etablierten Akteure, insbesondere Wohlfahrtsverbände, vermehrt auf die Umsetzung innovativer Konzepte angewiesen. Vor diesem Hintergrund ist davon auszugehen, dass nicht nur von SEs Innovationen vorangetrieben werden, sondern sich auch innerhalb etablierter Strukturen innovative Projekte entwickeln können.

1 Allerdings haben sich auch hier Nischenbereiche ausgebildet, in denen durchaus neue Akteure auftreten, wie z. B. spezialisierte Einrichtungen der stationären Jugendhilfe. 
Vor diesem Hintergrund soll daher zunächst eine begriffliche Klärung des schillernden Begriffs „Social Entrepreneurship“ vorgenommen werden (2) und ein analytischer Rahmen für die empirische Annäherung an das Feld skizziert (3) und das methodische Vorgehen umrissen werden (4). Ausgehend von der entwickelten Arbeitsdefinition wird anhand von zwei Handlungsfeldern (der Förderung von Kindern mit Migrationshintergrund in Grund- und Förderschulen sowie der kultursensiblen Altenhilfe und Altenpflege) das Spektrum sozialunternehmerischer Aktivitäten in Deutschland aufgezeigt und systematisiert. Hierfür wird auf qualitative und quantitative Daten zurückgegriffen, die im Rahmen empirischer Untersuchungen in den beiden Sektoren Altenhilfe und Jugendhilfe erhoben wurden(5.)Im abschließenden Fazit werden die Implikationen unserer Befunde diskutiert und die Relevanz und das Innovationspotential des Phänomens der Social Entrepreneurs in einem etablierten Wohlfahrtsstaat eingeordnet (6.).

\section{2. "Social Entrepreneurship": Konturen eines schillernden Begriffs}

Unter dem Motto „Everyone can change the world“ (Bornstein 2007; Elkington/ Hartigan 2008; Bornstein/Davis 2010) ist "Social Entrepreneurship" (im weiteren SE) in den letzten Jahren zum Hoffnungsträger einer Reaktivierung des Sozialen, einer Versöhnung von Unternehmertum und Gemeinwohl stilisiert worden. Diskursgeschichtlich stellt es das aktuelle „Gegenmodell“ zu den als defizitär und verkrustet dargestellten etablierten Strukturen der Wohlfahrtsproduktion dar. Nach dem tendenziellen Abflauen der Begeisterung für das Modell der Bürgeroder Zivilgesellschaft ist mit SE ein neuer Topos auf den akademischen Markt getreten. Im Mittelpunkt stehen dabei nicht die brachliegenden Ressourcen der Gesellschaft, sondern die Innovationskraft und das Engagement von Einzelpersönlichkeiten. Ausgewählte Gründerpersönlichkeiten werden insbesondere von den Mittlerorganisationen Ashoka und Schwab Foundation in Szene gesetzt und im Rahmen der aufkommenden SE Literatur diskutiert (vgl. die Beiträge in Háckenberg/Empter 2011; Jähnke et al. 2011).

Bislang hat sich in Deutschland keine einheitliche Definition des aus dem angelsächsischen Bereich stammenden Begriffs des "Social Entrepreneurship“ durchsetzen können. Die simple Übersetzung aus dem Englischen (,Sozialunternehmertum") ist ebenso wenig eindeutig wie die häufig anzutreffende Gleichsetzung des Begriffs mit (vermeintlich) philanthropischen Gründerpersönlichkeiten. Neben der Abhängigkeit vom jeweiligen institutionellen Kontext variieren die Interpretationen dessen, was unter SE zu verstehen ist, mit der wissenschaftlichen 
Disziplin, aber auch mit der Motivation, Message oder Handlungsorientierung des den Begriff verwendenden Autors. ${ }^{2}$

Insbesondere in der betriebswirtschaftlichen Entrepreneurship-Forschung wird SE als Teilphänomen von Unternehmertum erfasst (vgl. Perrini/Vurro 2006). Vielfach fokussiert SE auf die Erfassung des einzelnen, förderungswürdigen Social Entrepreneurs. Diese starke Fokussierung auf den einzelnen Initiator bzw. Handelnden geht von einer hervorstechenden Stellung einer Einzelperson (ggf. innerhalb einer Organisation) aus. Entweder hat sie die Erschließung eines neuen Handlungsfelds initiiert oder eine neue, den herausragenden Erfolg der Organisation begründende Herangehensweise an ein gesellschaftliches Problem eingeführt. Der in diesem Sinne verstandene Social Entrepreneur findet seine Motivation in dem Willen, eine drängende gesellschaftliche Frage zu bearbeiten. Ein solches Verständnis wird beispielsweise der Förderung durch ASHOKA zugrunde gelegt (vgl. Ashoka 2010). Meist ist das Engagement des Social Entrepreneurs biographisch erklärbar. Die zumindest teilweise vorhandene Risikobereitschaft begründet sich hier aus einer philanthropischen Haltung. Diese Fokussierung und Personalisierung mag im Hinblick auf eine Lenkung der medialen Aufmerksamkeit zweckmäßig sein, begrenzt allerdings den Untersuchungsgegenstand auf eine äußerst kleine Gruppe von Akteuren, deren Eruierung infolge der subjektiven Kriterien übermäßig von der Selbstwahrnehmung der Einzelperson abhängig wäre. Ausgehend von den Teilbegriffen ,Social' und ,Entrepreneurship' umfasst SE in Deutschland u. E. jedoch ein sehr viel weiteres Akteursspektrum und ein breites Angebotsfeld (vgl. auch Heinze et al. 2011). Als „social“ können solche „entrepreneurships" bezeichnet werden, die in zweifacher Weise gesellschaftliche Relevanz aufweisen: hinsichtlich ihres Aufgabenspektrums und ihrer Ausstrahlungskraft in die Gesellschaft. Der Tätigkeitsbereich sollte insofern Dienstleistungen umfassen, die sich an Menschen in besonderen Problemlagen bzw. mit besonderen Hilfebedarfen richten und dazu dienen, diese Problemlagen zu reduzieren und Hilfebedarfe zu befriedigen(vgl. Heinze/Naegele 2010).

Die den SE unterstellte Gemeinwohlorientierung äußert sich in den realisierten Effekten und ist konstitutives Element der jeweiligen Unternehmenskultur. Der Begriff des „Unternehmerischen“ (,Entrepreneurship“) kann im organisationssoziologischen Sinne als innovationsorientiertes, strategisches und seine Risiken selbst verantwortendes Handeln von Organisationen verstanden werden.

2 Aber auch im angelsächsischen Raum ist der Begriff alles andere als eindeutig. Die unterschiedlichen Autoren setzen dabei auf sehr unterschiedliche Schwerpunkte dic zu variablen Implikationen bezüglich der Untersuchungseinheit, der Rechtsform, der Finanzierung und der Eigentümerstruktur führen (Dees/Anderson 2006; Kerlin 2006; Defourny/Nyssens 2010; Teasdale 2012). 
Unternehmerisches Handeln zeichnet sich demnach erstens durch seine strategische Orientierung aus: Es ist in Abgrenzung zu bürokratischem Handeln an Zielen und Ergebnissen orientiert und nicht an einer ,Abarbeitung'vorgegebener Aufgaben. Strategisches Handeln heißt auch die Nutzung von Verfahren und Instrumenten, die es ermöglichen, rechtzeitig auf ein Nichterreichen der Ziele zu reagieren (Management). Zweites Kennzeichen unternehmerischen Handelns ist die Innovationsorientierung. Ziel dieser Orientierung ist es, neue Handlungsfelder zu entdecken und zu bearbeiten oder bekannte Felder (durch Evaluation) neu anzugehen(vgl. Kirzner 1999). Drittes Kennzeichen ist die Verantwortlichkeit für mögliches Scheitern, also eine Internalisierung des eigenen Geschäftsrisikos. Die Inkorporierung dieser Handlungsorientierung in eine Organisation mit einer im weiteren Sinne „sozialen“ Aufgabe stellt also ein zentrales Kennzeichen von SE dar.

Die Thematisierung der Verknüpfung von unterschiedlichen Handlungsorientierungen innerhalb einer Organisation ist nicht neu. So greift bspw. das Konzept des „Dritten Sektors“ (Salamon/Anheier 1996) aber auch des „Intermediären Raums“ (Evers 1995) gerade solche Organisationen auf, die sich keinem der drei Sektoren „Markt“, „Staat“ oder „Gemeinschaft“ eindeutig zuordnen lassen, sondern vielmehr Handlungsorientierungen und Steuerungsphilosophien aller drei Sektoren integrieren. Zu diesen „Dritte-Sektor-Organisationen“ werden auch die deutschen Wohlfahrtsverbände gezählt. Durch die veränderten institutionellen Kontexte in Richtung von Vermarktlichung und Professionalisierung hat sich die Position der Wohlfahrtsverbände innerhalb dieses intermediären Raumes verändert und ausdifferenziert. Zwischen den einzelnen organisatorischen Ebenen innerhalb eines Verbandes aber auch zwischen operativen Einheiten auf einer Ebene bestehen zum Teil erhebliche Unterschiede in Bezug auf die Balance zwischen marktlichen, hierarchischen und solidarischen Handlungsorientierungen. Dabei ist nach einer Phase der Schwerpunktsetzung auf eine stärkere Marktorientierung erkennbar, dass einige Organisationen aufgrund des entstehenden Legitimationsdefizits (Schneiders 2010) zunehmend auf Elemente der solidarischen Einbettung abheben. Insgesamt kann konstatiert werden, dass der soziale Dienstleistungssektor und die ihn konstituierenden Organisationen durch eine zunehmende Hybridität (Evers/Ewert 2010) geprägt sind.

$\mathrm{Zu}$ diesen hybriden Organisationen können auch die o. g. Social Entrepreneurs gezählt werden. Es stellt sich daher die Frage, ob es sich hierbei um eine originär neue Organisationsform handelt und ob Social Entrepreneurs neue Formen der Produktion sozialer Dienstleistungen entwickeln, die gegenüber den etablierten Trägern über spezifische Vorteile verfügen und ggf. zu einer Lösung des Dilem- 
mas von zunehmenden Bedarfen bei gleichzeitig stagnierenden öffentlichen Ressourcen beitragen können. SE bewegt sich dabei (ebenso wie andere Organisationen des „Dritten Sektors“) zwischen den Polen einer marktgetriebenen - auch gewinnorientierten - Orientierung, einer an gemeinschaftlichen Werten orientierten Perspektive und einer auf das ,große Ganze“ gerichteten ,staatsorientierten " bürokratischen Rationalität. SE weisen - so die These - eine besondere Form des Mischungsverhältnisses dieser drei Handlungsorientierungen auf. „Hybridity is not therefore any mixture of features from different sectors, but according to this view, is about fundamental and distinctly different governance and operational principles in each sector"(Billis 2010: 3 sowie die Beiträge in Brandsen et al. 2010).

SE können daher, müssen aber keine Non-Profit-Organisationen im Sinne des "Dritten Sektors" sein, da sie entgegen der von Salamon/Anheier (1996: $125 \mathrm{ff})$ entwickelten Kriterien für Dritte Sektor Organisationen zumindest zeitweise keine formale Struktur aufweisen und neben einer Gemeinwohl- auch eine Profitorientierung aufweisen können. Dabei ist darauf hinzuweisen, dass die der John-Hopkins-Studie zugrunde liegende Definition von Dritte Sektor Organisationen als Residualkategorie (weder eindeutig „Markt" noch eindeutig „Staat") der tatsächlichen Struktur und Ausgestaltung der unter diesem Label zusammengefassten Organisationen immer weniger gerecht wird (vgl. zu UK und USA die Beiträge in Billis 2010). SE kann nicht nur in Bezug auf die Integration verschiedener Handlungsorientierungen als hybrid bezeichnet werden, auch die legale Verfasstheit kann keiner Rechtsform eindeutig zugeordnet werden. So ist SE nach der oben skizzierten Definition sowohl als Einzelunternehmen, Stiftung, gemeinnütziger Verein/Verband als auch in Form von Public Private Partnership denkbar. Innerhalb eines solchen ,neuen“ SE-Sektors könnten sehr unterschiedliche Organisationsformen verortet werden. Das Spektrum würde dabei von der unternehmensnahen Stiftung mit einem Budget von mehreren Mio. Euro und dem Mitarbeiterstab eines Konzerns über die Initiative eines Einzelnen, der sich eines von ihm als drängend empfundenen sozialen Problems annimmt und dies zunächst als Einzelunternehmer ohne weitere Mitarbeiter bearbeitet, reichen.

Zusammenfassend gehen wir also von einem auf einen spezifischen „Handlungsstil", nicht auf eine spezifische Organisationsform gerichteten Begriff von Social Entrepreneurship aus, was dem Begriff -so man ihn ernst nehmen will - u. E. eher gerecht wird. Belastbare Daten zur Zahlder Organisationen, die diesem SE Sektor zugerechnet werden können sowie den von ihnen bearbeiteten Tätigkeitsfeldern, sind bislang jenseits der eindeutig dem privat-gewerblichen bzw. Marktsektor zuzuordnenden Unternehmen, für den auch amtliche Statistiken vorliegen, nur in unsystematischer Form vorhanden. Dies ist vor allem auf die Hy- 
bridität der Organisationen zurückzuführen, die eine eindeutige Zuordnung erschwert. Der hier interessierende Sektor des „Social Entrepreneurship“ im hier verwendeten weiteren Sinn wird in Deutschland aber um ein Vielfaches größer sein, als die bei den beiden Organisationen Ashoka und Schwab-Foundation akkreditierten 40 bzw. 11 Fellows bzw. Social Entrepreneurs (Stand Juli 2012). Diese These soll anhand einer empirischen Untersuchung in zwei sozialpolitischen Handlungsfeldern überprüft werden, dessen analytischer Rahmen im Folgenden dargestellt wird.

\section{Social Entrepreneurs als hybride Organisationen: analytischer Rahmen}

In der Literatur zu „Social Entrepreneurship“ lassen sich zwei wesentliche Perspektiven identifizieren: Während „Essentialisten“ auf SE als eigenen Typus fokussieren und sie in einem engen Sinne gemäß dem Mainstream-SE-Diskurs als distinktes Phänomen identifizieren (vgl. Jansen et al. 2010), betrachten „Subsumisten" SE als Sonderfall von Organisationen der sozialen Leistungsproduktion, die sich durch verschiedene Mischungsverhältnisse der Spezifika von Markt, Gemeinschaft und Staat auszeichnen und sich eher durch ein "mehr und weniger" als ein „entweder-oder" kennzeichnen(vgl. Heinze et al. 2011). Aus letzterer Perspektive interessieren eher spezifische Mischungsverhältnisse und die Einbettung in das Ensemble anderer Träger der Wohlfahrtsproduktion. Dies wird umso relevanter, möchte man den SE Begriff im internationalen Vergleich nutzbar machen. Es ist darauf hinzuweisen, dass sich der SE Diskurs zuerst in Entwicklungsländern etablierte und im Anschluss auf die „,defizitären“/,defekten“ Wohlfahrtsstaaten des angelsächsischen Raumes ausbreitete (vgl. Nicholls 2006; Defourny/Nyssens 2010). Eine Übertragung des Konzepts auf die hinsichtlich sozialer Dienste „dichter besiedelten" kontinentaleuropäischen oder gar skandinavischen Verhältnisse muss daher vorhandene Strukturen und Akteure, mithin ihre institutionelle Einbettung berücksichtigen und ihre eventuelle Disparität von anderen Organisationen des Dritten Sektors herausarbeiten (vgl. Kerlin 2012).

Im Folgenden wird das Spektrum von Akteuren und Organisationsformen ausgelotet, die einem ,weiten Begriff" des SE zuzuordnen sind. SE lässt sich, in Anlehnung an die oben entwickelte Arbeitsdefinition, in vier Dimensionen operationalisieren. Diese Dimensionen schaffen den Analyserahmen, um die vorzufindenden Organisationsstrukturen zu verorten und einen empirischen Einblick in das diffuse Feld zu erlauben.

Eine erste Unterscheidung betrifft den Innovationsgrad: Stellen die Angebote tatsächlich an professionellen Standards orientiert „neue“ Dienstleistungen 
zur Verfügung oder werden nur etablierte Verfahren mit neuen Begrifflichkeiten versehen? Zum zweiten wird der Impuls für die Initiierung des Angebotes bzw. Projektes untersucht: Werden die Organisationen von neuen Akteuren gegründet („Entrepreneurs") oder entwickeln sie sich aus etablierten Institutionen heraus („Intrapreneurs“). Damit verbunden ist die dritte Dimension: Entsprechen die realen SEs dem in der euphorischen SE-Literatur häufig kolportierten Einzelkämpfer (z. B. Elkington/Hartigan 2008) oder bewegen sie sich innerhalb etablierter Netzwerke? Viertens werden die internen Prozesse der Organisationen betrachtet: Orientiert sich die Steuerung der Organisationen eher an bürokratischen Routinen oder an Prinzipien eines strategisch orientierten Managements? Tabelle 1 bietet einen Überblick über die Operationalisierung der Analysedimensionen.

Tabelle 1: Analysedimensionen

\begin{tabular}{|c|c|c|}
\hline Pol 1 & Dimension & Pol 2 \\
\hline Innovator & $\begin{array}{c}\text { Innovationsgrad } \\
\text { Innovative organisatorische } \\
\text { Strukturmerkmale } \\
\text { Neue inhaldiche Schwerpunkte }\end{array}$ & Inkrementalist \\
\hline Entrepreneur & $\begin{array}{c}\text { Impuls } \\
\text { Gründung außerhalb und unabhângig von } \\
\text { bestehenden Organisationen }\end{array}$ & Intrapreneur \\
\hline Einzelkāmpfer & $\begin{array}{c}\text { Vernetzung } \\
\text { Bestehende Kooperationsstrukturen } \\
\text { Kooperationsbereitschaft }\end{array}$ & $\begin{array}{l}\text { Vernetzungs- } \\
\text { stratege }\end{array}$ \\
\hline Manager & $\begin{array}{c}\text { Steuerung } \\
\text { Nutzung betriebswirtschaftlicher } \\
\text { Instrumente } \\
\text { Dokumentation }\end{array}$ & Bürokrat \\
\hline
\end{tabular}

Die Messung der Dimensionen von SE stellt methodisch eine Herausforderung dar. Zur Ermittlung der Innovationskraft der Akteure haben wir die Innovativität der angebotenen Projekte anhand ihrer Strukturmerkmale und inhaltlichen Schwerpunktsetzung bewertet (vgl. Kapitel 5.1). Ausgehend von der These, dass innovative Projekte auch von Akteuren innerhalb etablierter Strukturen initiiert werden, wurde nach den Motivationen für die Initiierung und der Organisationsform des Projektes gefragt. Ein weiteres zentrales Element von SE ist die Vernetzung mit anderen im Feld aktiven Akteuren. Zur Ermittlung des Vernetzungsgrades wurden bestehende Vernetzungen,Vernetzungsbereitschaft und Kooperation 
im Rahmen der Projekte erfasst. Die Steuerung bzw. Strategiefähigkeit wurde durch Fragen zur Nutzung betriebswirtschaftlicher Methoden, sowie Durchführung von Evaluationen/Dokumentation operationalisiert.

Die Auswahl der sozialpolitischen Handlungsfelder orientierte sich an zwei in der klassischen Entrepreneurship-Literatur diskutierten „Idealtypen“ des Unternehmers, dem Schumpeterschen Innovator und dem Kirznerschen Nischenentdecker (vgl. Kirzner 1999). Während der erstere tatsächlich Neuerungen erfindet und einführt, entdeckt Kirzners Unternehmer brachliegende Nischen als Betätigungsfeld und nutzt so Gelegenheiten. Je dichter das Feld bestehender Angebote bestellt ist, desto weniger Lücken tun sich auf, die unternehmerisches Handeln des anspruchsloseren Typs zulassen. Daher ist davon auszugehen dass in etablierten Wohlfahrtsstaaten, der Unternehmertypus und die Bedeutung von Sozialunternehmern anders gelagert sind. Während das Handlungsfeld der Förderung von Kindern mit Migrationshintergrund in Primarschulen ${ }^{3}$ eher dem innovatorischen Typ in einem schon etablierten Feld zugeordnet werden kann, handelt es sich bei der kultursensiblen A:ltenhilfe ${ }^{4}$ um ein zwar stark expandierendes Handlungsfeld, aber quantitativ betrachtet noch um eine Nische innerhalb der Altenhilfe und-pflege insgesamt.

\section{Forschungsdesign}

Um die Frage nach der Rolle von Social Entrepreneurs im deutschen Falleines etablierten Wohlfahrtsstaats zu untersuchen, wurden zunächst innovative Projekte in den jeweiligen Handlungsfeldern identifiziert und anschließend hinsichtlich ihrer Strukturen und Handlungsstile untersucht. Durch diese Vorgehensweise konnten sowohl innovative Projekte von etablierten als auch von neuen Akteuren erfasst werden. Auf der Grundlage unserer These, dass der Spielraum für Social Entrepreneurs von der Dominanz der etablierten Strukturen abhängig ist, wurden für die konkrete Umsetzung der Untersuchung die Altenhilfe und Altenpflege und die Kinder- und Jugendhilfe ausgewählt. Ausgelöst durch eine sich ausbreitende

3 In den letzten Jahren haben zahlreiche internationale Schulleistungsuntersuchungen wie z.B. die PISA und IGLU Studien gezeigt, dass Kinder und Jugendliche mit Migrationshintergrund in Deutschland in den verschiedenen Leistungsbereichen signifikant schlechter abschneiden als Schüler/-innen ohne Migrationshintergrund. Dieser Leistungsrückstand erfordert eine gezielte Förderung insbesondere der sprachlichen Fähigkeiten der Kinder und Jugendlichen mit Migrationshintergrund (vgl. Gogolin et al. 2003).

4 Kultursensible Altenhilfe und -pflege „hat zum Ziel, pflegebedürftigen Menschen eiri Leben mit ihren je eigenen kulturellen Prägungen und Bedürfnissen zu ermöglichen (Arbeitskreis Charta für eine kultursensible Altenpflege 2002: 26). In der Praxis fokussieren die Projekte auf ältere Menschen mit Migrationshintergrund, die in Deutschland leben. 
Ökonomisierung ist der Bereich der Altenhilfe- und Altenpflege durch eine zunehmende Öffnung für privat-gewerbliche Anbieter geprägt (Schneiders 2010). Dahingegen lässt sich im Bereich der Kinder- und Jugendhilfe eine starke Persistenz der Trägerstrukturen gegenüber Privatisierungs- und Ökonomisierungsentwicklungen beobachten(Grohs 2010).

Das methodische Vorgehen der hier vorgestellten empirischen Untersuchung basiert auf einem Mixed-Method-Design, das quantitative und qualitative Methoden verschränkt. In einem ersten Schritt wurde in beiden Handlungsfeldern eine Online-Befragung durchgeführt, deren primäres Ziel die Identifizierung innovativer Projekte war. In einem zweiten Schritt wurden auf der Grundlage dieser Befragung für jedes Handlungsfeld zwei Fallstudien zur Validierung und Vertiefung der Ergebnisse der quantitativen Studie ausgewählt, die typischen theoretischen Merkmalen entsprechen.

Die Identifizierung von Social Entrepreneurs wird durch die disperse bzw. hybride Organisationsstruktur dieser "neuen“ Akteure erschwert. Daher war die Auswahl der Untersuchungseinheiten von erheblicher Bedeutung. Im Bereich der Bildungsförderung von Kindern mit Migrationshintergrund spielen die Grundund Förderschulen eine zentrale Rolle, da sie in den Schulgesetzen der Bundesländer als originäre Aufgabe u. a. mit der Förderung dieser Kinder beauftragt werden. Ausgehend von der These, dass ein Großteil der Projekte in Kooperation bzw. mit Wissen der Schulen durchgeführt werden, haben wir zur Identifizierung innovativer Projekte neuer und etablierter Akteure die Schulleitungen aller deutschen Grund- und Förderschulen befragt. Im zweiten Handlungsfeld wurde mit der Befragung von Einrichtungen im Bereich der Altenhilfe und Altenpflege eine analoge Vorgehensweise gewählt - hier haben wir mangels Informationen zur Grundgesamtheit zur Datengewinnung ein kumulatives SamplingVerfahren(Schneeballverfahren) angewendet. Befragt wurden auf Grund der besseren Identifizierbarkeit also nicht SEs als Solche, sondern typische Ankerinstitutionen, an denen Projekte von SEs ansetzen. Die Fallstudien wurden auf der Grundlage der Online-Befragung nach den Kriterien inhaltliche und organisatorische Innovativität ausgewählt. Im Rahmen der Fallstudien wurden zehn Interviews geführt. Befragt wurden neben Schulleitungen und Leitern/-innen von Einrichtungen bzw. Projekten, Mitarbeiter/-innen sowie die jeweiligen Kooperationspartner und Finanziers der Projekte. 


\section{Ergebnisse}

Im Weiteren werden auf Grundlage dieser Daten die von uns identifizierten Projekte und Einrichtungen hinsichtlich ihrer Innovativität, ihrer Initiierung, der Ausgestaltung von Kooperationsbeziehungen, der strategischen Ausrichtung, den Finanzierungsquellen und gesellschaftliche Rahmenbedingungen analysiert.

\subsection{Identifizierung von innovativen Projekten}

Im ersten Handlungsfeld der Förderung von Kindern mit Migrationshintergrund (Kids) wurden bundesweit die Schulleitungen von 15.238 Grund- und Förderschulen per Mail angeschrieben. Von den 1.605 Schulen, die an der Befragung teilgenommen haben(dies entspricht einer Rücklaufquote von $11 \%$ ), gaben 821 Schulen an, dass Projekte für Kinder mit Migrationshintergrund angeboten werden. Der inhaltliche Schwerpunkt dieser Projekte liegt in über $90 \%$ der Fälle auf der sprachlichen Förderung der Schüler/-innen (sowohl der deutschen als auch der Muttersprache).

Unter Einbeziehung der von den Schulleitungen zur Verfügung gestellten Projektbeschreibungen wurden auf der Grundlage eines Gutachtens der BundLänderkommission zur Förderung von Kindern und Jugendlichen mit Migrationshintergrund (vgl. Gogolin et al. 2003)Indikatoren zur Bewertung der Innovativität der Angebote gebildet. Die Projekte wurden hinsichtlich ihrer Innovativität anhand folgender Indikatoren überprüft: inhaltliche Ausrichtung, Arbeitsform, Zielgruppe und zeitliche Dimension. Dementsprechend wurden folgende Ausprägungen der Kategorien als innovativ betrachtet:

1. Inhaltliche Ausrichtung: Projekte zur Sprachförderung der deutschen und der Muttersprache sowie Projekte zur Förderung der interkulturellen Fähigkeiten.

2. Arbeitsform: Projekte, die in einer Mindestfrequenz (mehr als einmal wöchentlich) angeboten werden.

3. Zielgruppe: Angebote, die die Eltern der Kinder mit einbeziehen.

4. Zeitliche Dimension: Projekte, die nach der Grundschule in der weiterführenden Schule weitergeführt werden oder aus dem vorschulischen Bereich (Kindergarten/Kindertagesstätte/Vorschule fortgeführt werden (ganzheitliche Konzepte).

Insgesamt konnten auf der Basis dieser Innovationskriterien 152innovative Projekte $(26 \%)$ identifiziert werden. Die weiteren 444 Projekte werden im Folgenden als ,traditionelle Projekte“ bezeichnet. 
Im zweiten Handlungsfeld der kultursensiblen Altenhilfe und Altenpflege (Care) wurden im Rahmen eines Schneeball-Sampling Verfahrens 1.099 Einrichtungen im Bereich der Altenhilfe und Altenpflege angeschrieben. 55 Einrichtungen der 155, die an der Befragung teilgenommen hatten, gaben an, dass sie ein Projekt im Bereich kultursensiblen Altenhilfe bzw, -pflege anbieten. Inhaltlich werden v. a. Beratungs- und Freizeitangebote, also Altenhilfe angeboten. Die Beurteilung der Innovativität in diesem Bereich stößt insofern an Grenzen, als in diesem noch relativ jungen Feld keine Indikatoren zur Bewertung vorliegen. Daher wurden die Befragten um eine subjektive Einschätzung der „Neuartigkeit" ihrer „Dienstleistungen“ gebeten. Auf der Grundlage dieser Einschätzung wurden 27 innovative und 21 traditionelle Projekte identifiziert.

Die Auswahl der Fallstudien erfolgte auch in diesem Handlungsfeld nach dem Kriterium der Innovativität. Tabelle 2 bietet einen Überblick über die ausgewählten Fallstudien ${ }^{5}$.

Tabelle 2: Öbersicht der Fallstudien in den Handlungsfeldern

\begin{tabular}{|c|c|c|c|}
\hline Fălle & Beschreibung & Akteure & Finanzierung \\
\hline Kids I & $\begin{array}{l}\text { Sprachförderung } \\
\text { unter Einbeziehung } \\
\text { der Eltern sowie von } \\
\text { Jugendlichen aus der } \\
\text { Zielgruppe }\end{array}$ & $\begin{array}{l}\text { Jugendförderverein, } \\
\text { Grundschule, Sparkasse, } \\
\text { Kommune }\end{array}$ & $\begin{array}{l}\text { Stiftungen(Sparkasse und } \\
\text { Aktion Mensch) }\end{array}$ \\
\hline Kids II & $\begin{array}{l}\text { Gymnasiasten unter- } \\
\text { richten Grundschüler }\end{array}$ & $\begin{array}{l}\text { etablierte Wohltātigkeits- } \\
\text { organisation, Grundschule, } \\
\text { Gymnasium }\end{array}$ & $\begin{array}{l}\text { Spenden und } \\
\text { Teilnahmegebühren }\end{array}$ \\
\hline Care I & $\begin{array}{l}\text { Sozialberatung und } \\
\text { Gruppenarbeit in } \\
\text { Länder- und län- } \\
\text { derübergreifenden } \\
\text { Gruppen }\end{array}$ & $\begin{array}{l}\text { Wohlfahrtsverband, eingebun- } \\
\text { den in lokales, themenbezoge- } \\
\text { nes Netzwerk (Runder Tisch) }\end{array}$ & $\begin{array}{l}\text { Eigenmittel, } \\
\text { kommunale } \\
\text { Aufwandsentschädigung } \\
\text { für Ehrenamtliche }\end{array}$ \\
\hline Care II & $\begin{array}{l}\text { Demenz-WG im GbR- } \\
\text { Modell }\end{array}$ & $\begin{array}{l}\text { Unternehmerin (ehem. } \\
\text { Mitarbeiterin eines } \\
\text { Wohlfahrtsverbandes), kommu- } \\
\text { nales Wohnungs-unternehmen, } \\
\text { Wohlfahrtsverband, } \\
\text { Migrantenorganisation, lokaler } \\
\text { Verein }\end{array}$ & $\begin{array}{l}\text { Stiftung des } \\
\text { Wohnungsunternehmens } \\
\text { und Pflegeversicherung }\end{array}$ \\
\hline
\end{tabular}

Quelle: eigene Darstellung

5 Die Fallstudien werden in diesem Aufsatz in anonymisierter Form dargestellt. Namen von Personen, Projekten, Organisạtionen und Orten wurden aus den Interviewauszügen entfernt. 


\subsection{Initiierung: Etablierte und neue Akteure}

Die Initiierung von innovativen Projekten kann durch verschiedene Akteure erfolgen. Unterschieden wird zwischen den im jeweiligen Handlungsfeld etablierten und „neuen“ Akteuren. Als Etablierte bezeichnen wir im Handlungsfeld der Förderung von Kindern mit Migrationshintergrund Schulen, Schulleitungen und Schulträger. Eltern (-initiativen), Fördervereine, Stiftungen und Unternehmen haben wir als „neue A kteure“ definiert. Im Feld der kultursensiblen Altenhilfezählen zu den etablierten Akteuren u.a. Träger oder Mitarbeiter/-innen von Wohlfahrtsverbänden, während Initiativgruppen oder Migrantenorganisationen als neue Akteure gelten. Als dritte Variante haben wir in beiden Handlungsfeldern auch die gemeinsame Initiierung durch etablierte und "neue" Akteure identifiziert.

Die Befragungsergebnisse der quantitativen Studie zeigen in beiden Handlungsfeldern eine Dominanz der etablierten Akteure (vgl. Abb. 1).

Abbildung 1: Initiierung von Projekten durch etablierte Akteure (in Prozent)

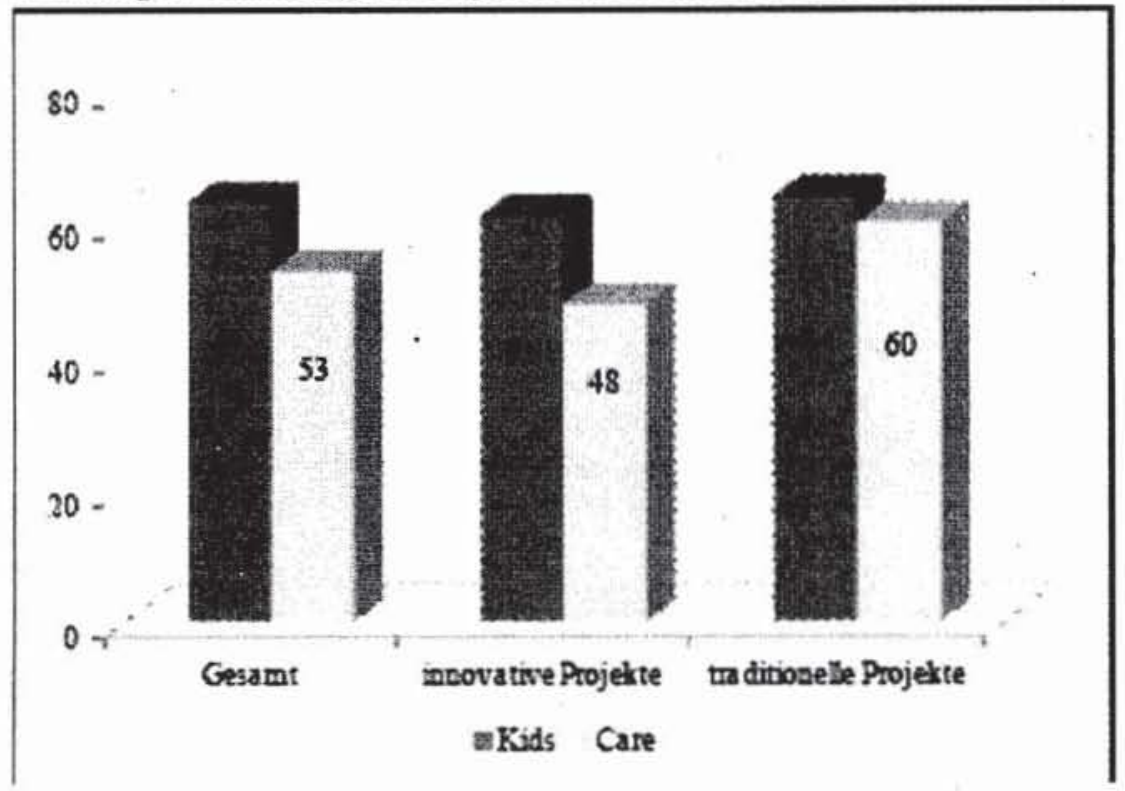

Quelle: eigene Darstellung (N(Kids): 582; N(Care): 47) 
Der Großteil aller Projekte insbesondere im Handlungsfeld der Förderung von Kindern mit Migrationshintergrundwird von etablierten Akteuren initiiert. In diesem Handlungsfeld zeigen sich in Bezug auf die Initiierung bzw. den Impuls zur Gründung nur minimale Unterschiede zwischen innovativen und traditionellen Projekten. Im Handlungsfeld kultursensibler Altenhilfe hingegen werden innovative Projektehäufiger von neuen Akteuren initiiert. Der Umkehrschluss, dass innovative Projekte ausschließlich von ,neuen“ Akteuren initiiert werden, ist jedoch nicht zulässig. Im Gegenteil: die Initiierungsaktivitäten der „neuen“ Akteure unterscheiden sich in diesem Handlungsfeld zwischen innovativen und traditionellen Projekten nicht. Abweichungen zeigen sich lediglich in der dritten Kategorie (gemeinsame Initiierung durch etablierte und „neue“ Akteure). Während von den traditionellen Projekten lediglich $5 \%$ durch eine Kooperation initiiert werden, sind über $22 \%$ der innovativen Projekte, durch eine gemeinsame Initiierung entstanden.

Die Fallstudien in den beiden Handlungsfeldern zeigen ebenfalls, dass etablierte Akteure eine entscheidende Rolle bei der Initiierung auch innovativer Projekte spielen. Darüber hinaus konnte unabhängig von der formalen Organisationsstruktur beobachtet werden, dass sowohl für die Initiierung als auch für die Umsetzung charismatische und engagierte Persönlichkeiten von Bedeutung sind (im Handlungsfeld der Förderung von Kindern mit Migrationshintergrund vor allem die Schulleitung). In zwei weiteren Fallstudien (Fallstudie Care II und Kids I) zeigte sich aber auch, dass etablierte Organisationen nicht immer genügend Spielraum für engagierte Mitarbeiter/-innen bieten. In diesen Fällen haben die Akteure zur Umsetzung ihrer Projektideen die etablierten Organisationen verlassen bzw. eine neue Organisation (z. B. Verein) gegründet. Das Gegenteil zeigte sich in Fallstudie Care I. Hier konnte ein innovatives Projekt in einer etablierten Organisation - in diesem Fall ein großer Wohlfahrtsverband - sowohl initiiert als auch umgesetzt werden.

Die Datenanalyse der quantitativen Untersuchung zeigt eindeutig, dass etablierte Akteure eine zentrale Rolle bei der Initiierung von innovativen Projekten spielen. Die Fallstudien haben dieses Ergebnis grundsätzlich bestätigt; zusätzlich scheinen erstens engagierte, motivierte Persönlichkeiten eine zentrale Rolle zu spielen und zweitens sollten etablierte Organisationen mehr Spielräume für engagierte Mitarbeiter/-innen schaffen, um innovative Projekt(ideen) innerhalb vorhandener Strukturen zu entwickeln. 


\subsection{Inkubatoren der Innovativität: Vernetzung und Kooperation}

Der Indikator der Vernetzung wurde auf zwei Ebenen untersucht. Zum einen auf der Ebene der Schule bzw. Einrichtung und zum anderen auf der Projektebene. Für die erste Ebene werden im Folgenden die Begriffe „Vernetzungsgrad“ und "Vernetzungsbereitschaft" verwendet, auf der Projektebene sprechen wir von „Kooperationen“.

Die Vernetzung und Kooperation ist grundsätzlich stark abhängig von den Persönlichkeiten, die in einer Organisation tätig sind, da Vernetzung und Kooperation ein hohes $\mathrm{Ma} ß$ an Engagement und Einsatzbereitschaft von den Akteuren erfordern. Die Schulen und Einrichtungen der kultursensiblen Altenhilfe sind nach eigenen Aussagen sehr gut in ihr (lokales) Umfeld eingebunden. Schulen weisen den höchsten Vernetzungsgrad zu anderen Schulen, der Kommunalverwaltung und zum Schulamt auf. Fast die Hälfte aller Schulen gibt an, dass sie in einem regelmäßigen Kontakt zu Sportvereinen und Kirchen steht. Darüber hinaus haben ein Viertel der Schulen Kontakte zu Jugendeinrichtungen und Unternehmen. Eher seltener sind Kontakte zu Stiftungen (13\%) oder Migrantenorganisationen (8\%).

„Wir haben verschiedene Kooperationen mit Institutionen vor Ort, mit der Musikschule, wir
haben uns als Powerschule zertifiziert, wir machen viel mit Ernährung und Bewegung und
Entspannung. Diese drei Säulen, dann haben wir ein Schulorchester zusammengebaut mit
Unterstützung des Fördervereins und der Musikschule vor Ort." (Interviewauszug Kids II)

Auch in den Einrichtungen der Altenhilfe und Altenpflege ist der Vernetzungsgrad sehr hoch. Über $80 \%$ der Einrichtungen geben an, regelmäßigen Kontakt zur Kommunalverwaltung und zu Wohlfahrtsverbänden zu haben. Aber auch mit Migrantenorganisationen, Kirchen und Initiativen stehen über die Hälfte der Einrichtungen in einem regelmäßigen Austausch. Ein geringerer Vernetzungsgrad besteht häufig zu Stiftungen ( $44 \%$ ) und Hochschulen (37\%). Den niedrigsten Vernetzungsgrad weisen die Einrichtungen zu Unternehmen auf (23\%).

Neben dem Vernetzungsgrad spielt auch die Bereitschaft zur Vernetzung eine zentrale Rolle. Diese Bereitschaft ist ein Indikator für die Aufgeschlossenheit von Institutionen gegenüber ihrem Umfeld. In beiden Handlungsfeldern lässt sich eine hohe Bereitschaft beobachten. Im Bereich der Altenhilfe und Altenpflege geben $89 \%$ der Einrichtungen an, dass sie gerne mit weiteren Institutionen kooperieren würden. Am häufigsten werden Stiftungen und Hochschulen genannt. Die Vernetzungsbereitschaft der Schulen ist mit $75 \%$ etwas geringer ausgeprägt. Die meisten Schulen wünschen sich vor allem Kontakte zu Kommunen, Stiftungen und Unternehmen. 
Auf Grund des hohen Vernetzungsgrades und der ausgeprägten Bereitschaft, Kooperationen einzugehen, verwundert es nicht, dass knapp jedes zweite der durch die Studie identifizierten Projekte in Kooperation angeboten wird. Die Unterschiede, die sich hierbei in Bezug auf das jeweilige Handlungsfeld ergeben, sind marginal. Deutliche Differenzen zeigen sich jedoch zwischen den innovativen und traditionellen Projekten. Innovative Projekte werden häufiger im Rahmen von kooperativen Projekten angeboten als traditionelle Projekte. Im Handlungsfeld der Förderung von Kindern mit Migrationshintergrund liegt die Differenz bei 7 Prozentpunkten, im Handlungsfeld kultursensibler Altenhilfe liegt die Differenz bei 28 Prozentpunkten. Dass Kooperationen wichtig sind für den Erfolg eines Projektes ist den Akteuren durchaus bewusst.

\footnotetext{
„Das ist jetzt mittlerweile auch in diesen ganzen Jahren was, wo ich mehr denn je überzeugt bin, also dass das einfach Sinn macht hier auch möglichst viele Kooperationen zu schaffen, ob das jetzt wir sind oder anderc. Dass man einfach diese unterschicdlichen Sichtweisen nutzen kann, einmal durch die Experten im System und dann aber auch nochmal von außen, die dann noch einmal ein anderes Spiel reinbringen und auch andere Handlungsmöglichkeiten und eigentlich war unsere Kooperation immer getragen von dem Gedanken, dass genau das Sinn macht." (Interviewauszug Kids I)
}

Dieses Zitat verdeutlicht, dass Kooperationen und Kooperationspartner die Qualität in Projekten steigern können. Unterschiedliche Erfahrungen, Ideen sowie das Wissen und die Kompetenzen der verschiedenen Akteure fließen in das Projekt ein und generieren auf diesem Weg einen Mehrwert.

In einem Projekt der kultursensiblen Altenhilfe (Fallbeispiel 2) zeigt sich, dass Kooperationen in manchen Fällen die Initiierung von Projekten erst ermöglichen.

\begin{abstract}
„Das war ebenso eine taktische Überlegung und dann haben wir erst an so eine türkische Demenz-WG gedacht. Sind viel rumgelaufen und haben mit Gott und der Welt geredet, aber dann gemerkt, die sind nicht so weit. Pardon... also die versorgen ihre dementiell erkrankten Menschen in der Familie... keine Ahnung. ..jedenfalls hatten wir da keinen Zugang. Und dann fielen uns die Russen, die auch eine sehr große Community hier sind, ein und das war dann ein bisschen einfacher also mit [Name des Vereins] haben wir kooperiert, die uns den Zugang geschaffen haben." (Intervicwauszug Care II)
\end{abstract}

Die Idee einer Wohngemeinschaft für Demenzerkrankte speziell für Menschen mit einem türkischen Migrationshintergrund aufzubauen, scheiterte an der fehlenden Nachfrage in der Zielgruppe. Durch die öffentliche Kommunikation der Projektidee und der damit verbundenen Probleme führte dazu, dass die Projektidee modifiziert wurde, die Angehörigen der Zielgruppe schließlich in die Umsetzung des Projektes mit einbezogen wurden und das Projekt nicht schon in der Anfangsphase scheiterte. In den Fallstudien spielte die Kooperation mit der Ziel- 
gruppe und deren Einbezug in die Angebotserstellung ganz grundsätzlich eine wichtige Rolle. Insgesamt wurden in drei der vier Fallstudien Menschen mit Migrationshintergrund nicht nur als Zielgruppe gesehen, sondern gezielt in die Umsetzung, Erbringung und Entwicklung des Angebotes mit einbezogen.

\subsection{Strategische Ausrichtung}

Die strategische Ausrichtung der Projekte wurde anhand von zwei verschiedenen Indikatoren operationalisiert: der Existenz von Dokumentationen und dem „Einsatz betriebswissenschaftlicher Instrumente“. Im Rahmen der quantitativen Befragung wurden verschiedene Dokumentationsformen abgefragt (vgl. Tab.3).

Tabelle 3: Verwendung verschiedener Dokumentationsformen nach Handlungsfeld

\begin{tabular}{lcc}
\hline & Kids & Care \\
\hline Pressebericht & $12 \%$ & $15 \%$ \\
regelmäBige interne Berichte & $41 \%$ & $36 \%$ \\
regelmäBige Berichte an Dritte & $15 \%$ & $25 \%$ \\
öffentliche Berichte & $7 \%$ & $13 \%$ \\
Evaluationen & $7 \%$ & $7 \%$ \\
unsere Arbeit wird nicht dokumentiert & $18 \%$ & $4 \%$ \\
\hline
\end{tabular}

Quelle: eigene Darstellung (N(Kids): 656; N(Care): 48)

In beiden Handlungsfeldern finden „regelmäßige interne Berichte" am häufigsten und Evaluationen am seltensten Anwendung. Damit findet ein Instrument am häufigsten Anwendung, das zwar standardisiert, aber mit geringem Informationsgehalt ausgestattet ist, wenn es um zukunftsgerichtetes Lernen oder Weiterentwicklung der Projekte geht.

Die Messung der Wirkung und die anschließende Anpassung der Prozesse und Projektziele machen die Evaluation zu einem Dokumentationsinstrument, das über den bloßen Beschreibungscharakter des Berichtes hinausgeht. In den Fallstudien im Handlungsfeld der Förderung von Kindern mit Migrationshintergrund zeigte sich jedoch eine grundsätzliche Skepsis im Bezug auf Evaluationen. Die Förderung von Kindern, unabhängig in welcher Form ist, nach Ansicht der Pädagogen ein vielseitiger und ganzheitlicher Prozess, der in einem Evalua- 
tionsverfahren nicht vollständig erfasst und somit die Entwicklung lediglich in verkürzter Form festhalten könne. Aber auch im Handlungsfeld kultursensibler Altenhilfe wird dieses Instrument der Wirkungsmessung nur selten angewendet, ganz im Gegensatz zur Dokumentation im Allgemeinen. In diesem Bereich geben lediglich $4 \%$ der Einrichtungen, dass ihre Arbeit überhaupt nicht dokumentiert wird, im Handlungsfeld Förderung von Kindern mit Migrationshintergrund ist dieser Anteil mit $18 \%$ deutlich höher, was auf eine weniger starke strategische Orientierung in diesem Handlungsfeld schließen lässt.

Zur Analyse des zweiten Indikators(,Einsatz betriebswirtschaftlicher Instrumente") wurde der Einsatz von Balanced Scorecards, Businessplänen oder Finanzplänen erfasst. Auf Grund der stärkeren Ökonomisierung und Finanzierungsabhängigkeit ist der Einsatz solcher Instrumente im Handlungsfeld kultursensibler Altenhilfe erwartungsgemäß häufiger anzutreffen. Immerhin $35 \%$ der Einrichtungen geben an, dass sie eines dieser Verfahren nutzen. Das Instrument der Finanzplanung findet dabei mit 20\% am häufigsten Anwendung. In der Förderung von Kindern mit Migrationshintergrund werden betriebswirtschaftliche Instrumente etwas seltener eingesetzt $(30 \%)$. Auch in diesem Handlungsfeld kommen Finanzpläne am häufigsten zum Einsatz. Diese sind insbesondere dann notwendig, wenn für das Projekt bei Dritten Mittel eingeworben werden müssen, was durch das folgende Zitat deutlich wird:

Und in diesem Kontext, als es darum ging, was ist eigentlich so, der Effekt, wenn man jahre-
lang so cine Arbeit gemacht hat, wie muss man das nochmal zusammenkriegen?Da ist dann
diese Idee entstanden und wir haben gesagt, OK, wir machen mal eine Kalkulation. Ich habe
dann zur Gabi gesagt, so jetzt mach du mal eine Konzeption und dann haben wir das Gerūst für
cine Antragsstellung crarbeitet. Und dann haben wir zusammen - weil ich da dic Erfahrungen
dazu hab-haben das natürlich auch gut unterfüttert und so und haben an die [Name der Stif-
tung] einen Antrag gesteltt und haben eine tolle Förderung gekriegt."(Interviewauszug Kids I)

Diese Indikatoren mit einer unternehmerischen Handlungsorientierung gleichzusetzen ist zumindest für die Akteure selbst nicht evident. In einem Interview mit zwei Mitarbeiterinnen einer der großen Wohlfahrtsverbände im Fallbeispiel Care I erhielten wir auf die Frage, ob sie im Rahmen des Projektes unternehmerisch handeln würden, folgende Antwort:

\footnotetext{
„Aber ich lasse mir jetzt auf dem Mund zergehen, das Stichwort „Unternehmerisches Handeln“. Und dann stelle ich mir einen Geschäftsführer vor, der sagt: „Wir schreiben dieses Jahr schwarze Zahlen“, oder vielleicht sagt er mir nāchstes Jahr: „Wir schreiben rote Zahlen." Das war jetzt nur eine kurze Ergebnisformulierung was sic da gesagt haben, aber natürlich muss ein Wohlfahrtsverband ein Träger unternehmerisch agieren." (Interviewauszug Care I)
}

Ein wenig später präzisiert sie dann: 
„Insgesamt agiert der Verband aber unternehmerisch, nicht gewinnorientiert, aber er hat Personalkosten und am Ende muss es eben aufgehen."(Interviewauszug Care I)

Im gleichen Interview wurden wenig später die offeneren Strukturen dieses Handlungsfeldes explizit, die hinsichtlich der Anbieterstrukturen durchaus als marktähnlich bezeichnet werden können:

\begin{abstract}
„Aber natūrlich ist es auch so, dass in dem Moment, wo ein Thema gesellschaftlich oder kommunal so hochgehoben wird, stehen natürlich auch andere Akteure auf der Matte und sagen, das machen wir jetzt auch und grundsätzlich finde ich das gut. Wir kãmpfen ja für ein freieres, größeres Angebot. Wir sehen den Bedarf und wollen mehr Unterstützung. Und nicht nur bei uns, aber eben auch bei uns. Manchmal hat man schon so ein bisschen das Gefühl, es entsteht ein Markt."(Interviewauszug Care I)
\end{abstract}

Ausgehend von diesem Zitat ließe sich das Projekt aus Fallstudie Care I nach der hier gewählten Definition durchaus als ein Beispiel für Social Entrepreneurship bezeichnen. Dieses in einem Wohlfahrtsverband initiierte und umgesetzte Projekt erfüllt alle Kriterien unseres breiteren Social Entrepreneurship Begriffs: das Projekt löst auf eine innovative Art und Weise ein soziales Problem, nämlich die Integration von älteren Menschen mit Migrationshintergrund, die Hauptressource sind ehrenamtliche Helfer, die selbst einen Migrationshintergrund haben und das Projekt wird in einer unternehmrisch handelnden Organisation umgesetzt.

Betrachtet man den Zusammenhang zwischen innovativen Projekten und der strategischen Ausrichtung, so zeigt sich in der Förderung von Kindern mit Migrationshintergrund, dass insbesondere die traditionellen Projekte, ausgehend von den zugrunde gelegten Indikatoren, eine stärkere strategische Ausrichtung aufweisen. Traditionelle Projekte werden im Vergleich zu den innovativen deutlich häufiger evaluiert. Eine solche Evaluation wird bei traditionellen Projekten in $12 \%$ der Fälle durchgeführt und somit mehr als doppelt so oft wie in innovativen Projekten ( $5 \%$ ). Auch Instrumente der unternehmerischen Betriebsführung finden in traditionellen Projekten mit $22 \%$ deutlich häufiger Anwendung als bei den innovativen. Hier sind es lediglich $14 \%$ der Projekte, in denen Instrumente der unternehmerischen Betriebsführung angewendet werden. ${ }^{6}$ Demnach stellt sich grundlegend die Frage, ob unternehmerisches und strategisches Handeln Handlungsorientierungen sind, die gerade in den etablierten Organisationen ihre Anwendung finden, bzw. die Frage danach, ob die Definition von „Social Entrepreneurship" an dieser Stelle zu kurz greift. Immerhin treffen die Eigenschaften „innovativ" und „strategisch" auch auf Projekte zu, die in der allgemeinen De-

$6 \quad$ Im Handlungsfeld kultursensibler Altenhilfe zeigen die Testverfahren keine signifikanten Unterschiede zwischen der strategischen Ausrichtung von innovativen und traditionellen Projekten. 
batte als das Gegenteil eines Social Entrepreneurs angesehen werden wie z.B. Projekte von Wohlfahrtsverbänden. Solche Projekte lassen sich unter der ,neuen “ Terminologie des „Social Intrapreneurs“ fassen. Das dritte Element der zugrunde liegenden Definition von ,unternehmerisch“ ist, dass Social Entrepreneurs ihr „Risiko selbst verantworten“ inwieweit dies auf die identifizierten innovativen Projekte zutrifft, zeigt sich im folgenden Abschnitt.

\subsection{Finanzierung}

Die Frage nach dem „Risiko“ soll anhand der Finanzierung der Projekte näher untersucht werden. Im Einzelnen wird dargestellt, welche Ressourcen für die Finanzierung der Projekte genutzt werden und inwiefern versucht wird, neue Finanzquellen zu erschließen. Abbildung 2 veranschaulicht, welche Ressourcen in den beiden Handlungsfeldern zur Finanzierung der Projekte herangezogen werden. Unterteilt sind die Angaben auch hier wieder in traditionelle und innovative Projekte. Es zeigt sich, dass in beiden Handlungsfeldern in fast allen Fällen ,eigene Mittel ${ }^{\text {"7 }}$ zur Finanzierung der Projekte herangezogen werden. Gleich häufig werden öffentliche Mittel und Mittel von Dritten für die Finanzierung von Projekten eingesetzt. Eher selten fließen selbst erwirtschaftete Mittel in die Projekte ein. Grundsätzlich weisen die beiden Handlungsfelder eine ähnliche Finanzierungsstruktur auf. Größere Unterschiede finden sich lediglich hinsichtlich des Einsatzes öffentlicher Projektmittel und Mitteln von Dritten. Auf letztere greifen Projekte aus dem Handlungsfeld kultursensibler Altenhilfe deutlich häufiger zurück als Projekte im Handlungsfeld der Förderung von Kindern mit Migrationshintergrund. Insgesamt zeigt sich, dass Projekte aus dem Feld kultursensibler Altenhilfe mehr Finanzierungsquellen heranziehen als die Projekte im Schulbereich. Während die Anzahl der Finanzierungsquellen im Bereich der Förderung von Kindern mit Migrationshintergrundzwischen den traditionellen und innovativen Projekten kaum differiert, zeigen sich in der kultursensiblen Altenhilfe deutliche Unterschiede. Für die innovativen Projekte werden im Schnitt mehr Finanzierungsquellen herangezogen als für die traditionellen Projekte.

$7 \quad$ Bei den Schulen handelt es sich um Mittel der Schule oder des Schulträgers und bei den Einrichtungen der Altenhilfe und Altenpflege um Mittel der Einrichtung oder des Trägers. 


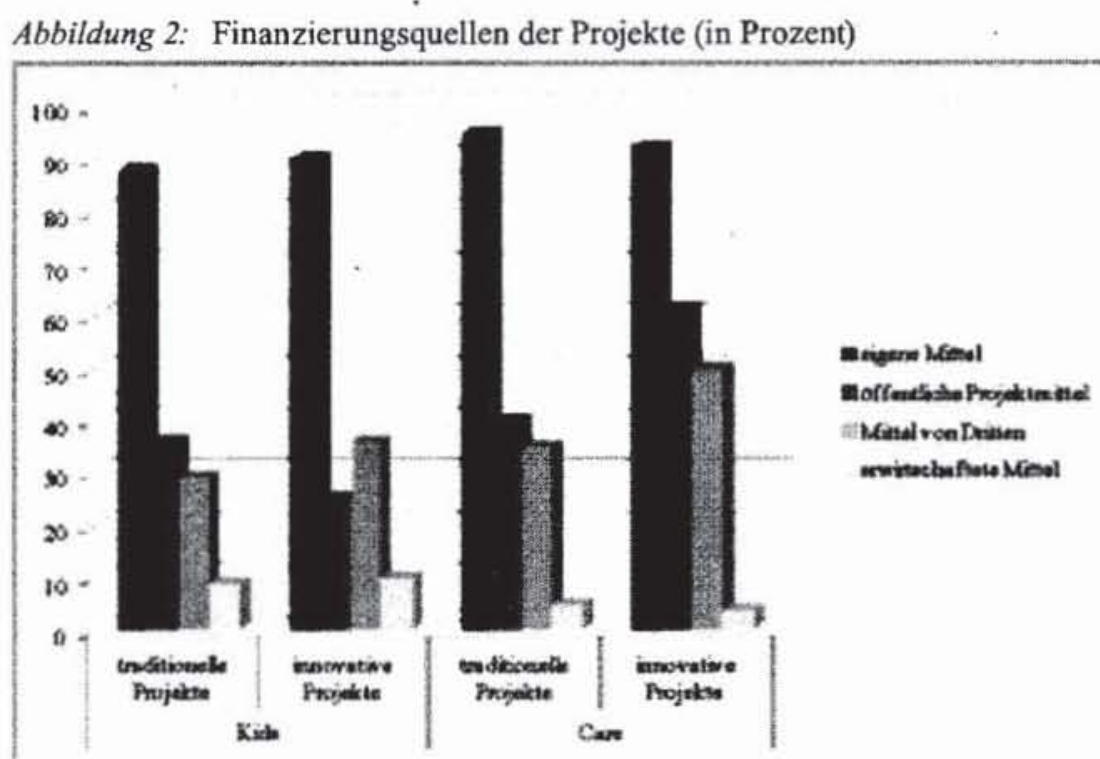

Quelle: eigene Darstellung (N(Kids): 568; N(Care): 46)

Vergleicht man die Finanzierungsstrukturen in den einzelnen Handlungsfeldern in Abhängigkeit von der Innovativität der Projekte, zeigt sich, dass für die innovativen Projekten weniger häufig öffentliche Projektmittel verwendet werden und dafür häufiger Mittel von Dritten, wie z.B. Stiftungen und Unternehmen. Im Handlungsfeld kultursensibler Altenhilfe zeigt sich hingegen, dass im Unterschied zu traditionellen Projekten der Einsatz von öffentlichen Mitteln und Mitteln von Dritten ansteigt, während es bei den anderen Finanzierungsquellen kaum zu Veränderungen kommt. In diesem Bereich scheint es bei den innovativen Projekten daher nicht zu einer Verschiebung nach dem Prinzip je-mehr-desto-weniger zu kommen, sondern einfach nur zu einem "mehr" an Finanzierungsquellen.

Das Thema Finanzierung wurde im Rahmen der Fallbeispiele noch einmal von einem anderen Blickwinkel aus betrachtet. Alle vier Fallbeispiele unterscheiden sich hinsichtlich ihrer Finanzierungsstrukturen.

Im Fallbeispiel Kids I wird die Finanzierung durch zwei Finanzquellen des Projektes sichergestellt: durch einen gemeinnützigen Verein und eine Stiftung. 
Das größte Problem, dass in diesem Projekt hinsichtlich der Finanzierung besteht, ist der begrenzte Finanzierungszeitraum.

„Haben wir jetzt gut genutzt, mit dem Projekt [Projektname] Das [Projektname] werden wir in einem anderen Setting, werden wir weitermachen. Die Frage ist, wie wird das finanziert [...]."(Interviewauszug Kids I)

Im Projekt Kids II, welches durch eine etablierte Wohltätigkeitsorganisation ins Leben gerufen wurde, wurde ein Verein gegründet, um die Finanzierung des Projektes sicherzustellen.

„Ja, das ist ein Verein, der ist also vom Finanzamt anerkannt, wir dürfen Spendenquittungen ausstellen, da kostet dic Mitgliedschaft zehn Euro im Jahr. Das ist ja nicht nennenswert."(Interviewauszug Kids II)

Neben den Mitgliedsbeiträgen für den Verein nutzt die Organisation weitere Quellen zur Spendenakquise und zur Mitteleinwerbung.

Ich muss kurz sagen, wir machen einmal im Jahr so ein Konzert, wo wir etwa so 8.000 bis
$10.000 €$ ubbrig behalten für das Projekt und einmal im Jahr nehmen wir an einem Weihnachts-
markt teil hier, in [Name der Stadt], [...], wo wir Glühwein ausschenken und wo wir auch
Wiehtel basteln. [...] Da nehmen wir dann auch nochmal irgendwie 5-6-7.000€ ein und dann
ist es so, dass die [...] Mitglieder, wenn die einen runden Geburtstag haben und sagen,.,Was
wünsche ich mir da, ich wünsche mir einfach, ich stell ein Sparschwein auf mit ciner Spen-
de für [Name des Projektes], und da kommen durchaus auch schon mal namenhafte Beträge
zusammen." (Interviewauszug Kids II)

Großen Wert legt die Organisation darauf, dass die Spenden den Gymnasiasten zu Gute kommen, die die Förderung für die Kindergarten- und Grundschulkinder übernehmen. Anderweitig anfallende Kosten versucht die Organisation auf anderen Wegen zu finanzieren.

„Das heißt, wir versuchen, wenn wir Prospekte drucken, da muss irgendein Unternehmen die Druckkosten übernehmen und alles Mögliche, was so an Sonderkosten entsteht, die möchte ich am liebsten, dass diese das Projekt nicht belasten, sondern das Geld wirklich cinsetzen und dann ist dic Spendenbereitschaft auch sehr groß, weil wir auch einen sehr groben Wirkungsgrad haben," (Interviewauszug Kids II)

Der große Vorteil dieser Organisation besteht dabei insbesondere in einem sehr gut ausgebauten Netzwerk in allen gesellschaftlichen Bereichen, so dass die Organisation ihren eigenen Ansprüchen im Rahmen des Projektes gerecht werden kann.

Im ersten Fallbeispiel im Feld kultursensibler Altenhilfe (Care I) ist die Finanzierung durch die Einbindung des Projektes in einen Wohlfahrtsverband sichergestellt. Darüber hinaus werden die Kosten des Projektes durch den Einsatz 
von Ehrenamtlichen möglichst gering gehalten. Nichts desto weniger handelt es sich um einen Zuschussbereich innerhalb des Verbandes, der von anderen Bereichen mitgetragen werden muss.

\begin{abstract}
.Wir sind ja hier in der Seniorenarbeit, kann ich ja mal sagen, wir sind ein Zuschussbereich. Innerhalb des Verbandes gibt es Bereiche, die sind auskömmlich finanziert, wir sind es als Seniorenarbeit nicht. Wir profiticren von - ich sage mal - den Kitas, dic wir haben oder von anderen Bercichen, wo eher am Ende 3,50 DM überbleiben." (Interviewauszug Care I)
\end{abstract}

Beim zweiten Fallbeispiel verfügt das an dem Projekt beteiligte Wohnungsunternehmen über eine eigene Stiftung, welche das Projekt (neben Bezügen aus der Pflegeversicherung) absichert. Absichert meint in diesem Fall, dass die aufgebaute Demenz-WG nur bei $100 \%$ iger Auslastung kostenmäßig tragfähig ist. Es kommt jedoch häufiger vor, dass Plätze vorübergehend frei sind und für diesen Fall können Mittel aus der Stiftung genutzt werden.

„Dieses GbR Modell schreckt dann doch viele und der Kostenfaktor, den wir über eine hausinterne Stiftung eventuell abfangen können, der ist bei anderen finanziell nicht darstellbar." (Interviewauszug Care II)

\title{
5.6 Die institutionelle Einbettung von Social Entrepreneurship
}

In einem abschließenden Analyseschritt ist der Frage nachzugehen, inwieweit sich die gesellschaftlich-institutionellen Rahmenbedingungen auf die Projekte auswirken, um die eingangs formulierten Fragestellungen nach den möglichen Nischen für Social Entrepreneurship in Deutschland zu überprüfen. Die Rahmenbedingungen werden in vier Kategorien gegliedert:

1. Rechtliche Rahmenbedingungen

2. Einfluss der Lokalpolitik

3. Einfluss des Trägers

4. Konkurrenz durch weitere/ähnliche Projekte

Die Analyse der vorliegenden quantitativen Befragungsdaten ergab, dass die Rahmenbedingungen sich im Großteil der Fälle weder fördernd noch hemmend auf die Initiierung oder Umsetzung der Projekte auswirken (vgl. Tab. 4). 
Tabelle 4: Auswirkungen von Rahmenbedingungen auf die Projekte

\begin{tabular}{lccc}
\hline Kids & fördernd & kein Einfluss & hemmend \\
\hline Rechtliche Rahmenbedingungen & & & \\
Lokalpolitik & $19 \%$ & $60 \%$ & $21 \%$ \\
Träger & $36 \%$ & $57 \%$ & $7 \%$ \\
Konkurrenz durch ähnliche Projekte & $26 \%$ & $69 \%$ & $5 \%$ \\
\hline Care & $6 \%$ & $90 \%$ & $6 \%$ \\
Rechtliche Rahmenbedingungen & $14 \%$ & $75 \%$ & $11 \%$ \\
Lokalpolitik & $61 \%$ & $39 \%$ & $0 \%$ \\
Träger & $85 \%$ & $13 \%$ & $2 \%$ \\
Konkurrenz durch ähnliche Projekte & $7 \%$ & $84 \%$ & $9 \%$ \\
\hline
\end{tabular}

Quelle: eigene Darstellung (N(Kids): 509; N(Care): 43)

Es zeigte sich in der Auswertung der quantitativen Studie, dass insbesondere die Schulen im Großteil ihrer Projekte keinen Einfluss der abgefragten Rahmenbedingungen wahrgenommen haben. Lediglich der Bereich der rechtlichen Rahmenbedingungen wurde von $20 \%$ der Schulen als hemmend empfunden. Im Bereich der kultursensiblen Altenhilfe wurde der Einfluss der Träger und der Lokalpolitik als eher fördernd wahrgenommen.

- Im Rahmen der qualitativen Falluntersuchungen konnten die Einflüsse der Rahmenbedingungen näher untersucht und konkretisiert werden. Dabei zeigte sich, dass in zwei der vier Fallbeispiele die etablierten Träger einen negativen Einfluss auf die Tätigkeiten und die Umsetzung neuer Projekte ausübten. Diese beiden Beispiele stammen aus je einem Handlungsfeld. Im Bereich der Förderung von Kindern mit Migrationshintergrund zeigte sich, dass in der öffentlichen Jugendhilfe bürokratische Strukturen die Umsetzung von Projekten hinauszögerten.

\begin{abstract}
„Man kann es sagen, wir sind ein Verein, der von den damaligen Mitarbeiterinnen der Jugendhilfe gegründet wurde, also weil das was man da in der Jugendhilfe, in der öffentlichen leisten konnte, an verschiedenen Stellen, nicht umsetzbar war. In den 70iger Jahren gab es ja dann auch diese Finanzgeschichten, ähnlich wie hier jetzt und da hat man natürlich auch gesagt. Die Jugendhilfe, die braucht da länger, um bestimmte Projekte und bestimmte Angebote einzubringen. Und das ist eigentlich so dieser Hintergrund." (Interviewauszug Kids I)
\end{abstract}

Die Lösung dieses Problems war die Gründung eines Vereins. In diesem Verein engagieren sich Mitarbeiterinnen und Mitarbeiter der öffentlichen Jugendhilfe 
ehrenamtlich. In diesem Verein können dann die Projekte umgesetzt werden, die sich in der öffentlichen Jugendhilfe nicht oder nur schwerer umsetzten lassen.

Auch im Handlungsfeld kultursensibler Altenhilfe stehen engagierte, motivierte Persönlichkeiten vor Problemen mit den etablierten Organisationen. In dem konkreten Fall handelt es sich um die ehemalige Mitarbeiterin eines Wohlfahrtsverbandes.

„Und ich hatte das unternehmerische Vorgehen vorgeschlagen, aber ich war auch keine Lei-
tung, ich war eine Mitarbeiterin und ich war überidentifiziert und ich sage mal unternehme-
risch, deshalb war ich sicherlich eine sehr problematische Mitarbeiterin, weil ich halt cben das
so weiterentwickeln wollte. Ich war sozusagen eher leitungsmäBig in meinem Kopf unterwegs
und habe dann den Konflikt mit der Geschäftsführung auf dic Spitze getricben, das kann man
als Mitarbeiter, das geht ja gut insbesondere dann, wenn man eine schwache Geschäftsfüh-
rung hat, dann geht das ganz gut. Und ich hatte sozusagen den Streit dahingehend fokussiert,
sozusagen. Ich war der Ansicht wir müssten die Beratungsstelle anders weiterentwickeln und
sowohl meine Leitung als auch meine Kollegen waren der Meinung dass das nicht so ist und
dann ist dic logische Konsequenz das sie entweder in die innere Kündigung gehen oder rich-
tig kündigen." (Interviewauszug Carc II)

Die Konsequenz war, dass diese Mitarbeiterin den Verband verlassen hat und ein eigenes Unternehmen gründete. Entstanden ist daraus die Idee, Demenz-WGs im GbR-Modell zu betreiben. In diesem Projekt lassen sich Innovationen nicht nur auf der Produkt-, sondern auch der Strukturebene identifizieren. Hinsichtlich der Kooperations- und Finanzierungsstrukturen erscheint dieses Projekt einzigartig, woraus jedoch eine Reihe weiterer Probleme entstanden sind.

„Unsere These wäre zunächst gewesen, dass man lokal arbeiten muss, um überhaupt solche Projekte zu crkennen, weil nur auf lokaler Ebene kann man schen, wo der Bedarf ist. Wir haben aber zunächst nicht daran gedacht, dass die Kommune so hinderlich scin kann." (Interviewauszug Care II)

In diesem Fallbeispiel wird die Kommune als hinderlich empfunden. Die Akteure sind frustriert, sie fühlen sich der Willkür der Kommune ausgesetzt. Wobei die Grenzen zwischen Kommune und rechtlichen Rahmenbedingungen verschwommen sind, was anhand des folgenden Zitates deutlich wird:

„Nur um das auf den Punkt zu-bringen, wir haben ja eine ausgeprägte Bürokratic in Deutschland, es ist ja bei uns im Projekt zentral, dass dic Stadt in vielen Projekten einfach dic Projekte behindert mit zu vicl Bürokratic. Das ist dann quasi auch hier der Fall. Wenn man uns fragen würde .,Was könnte die Stadt machen, um solche Projekte zu fördern." Dann würde ich sagen, dass wir gar keine aktive Unterstützung brauchen oder wollen, aber dass die zumindest nicht mit mehr Regulierung das Ganze verschlimmern." (Intervicwauszug Care II) 
Was genau die Interviewte unter „ausgeprägter Bürokratie“ versteht, zeigt das folgende Zitat:

„Wir sind ja ein Land, in dem alles organisiert wird. Die WGs werden jetzt andersrum angegriffen, weil jetzt schic Bt man ja mit dem vollen Brandschutz, den vollen Hygienestandards und so was alles auf diese armen kleinen Wohngemeinschaften." (Interviewauszug Care II)

Anhand dieses Interviewauszuges wird deutlich, dass in Deutschland rechtliche Strukturen vorherrschen, die Unternehmungen wie die Demenz-WG erschweren können und von den engagierten Akteuren als hinderlich zum Teil sogar als willkürlich empfunden werden.

Ein positives Beispiel für den Einfluss von Trägern und Kommune ist das Fallbeispiel I im Handlungsfeld kultursensible Altenhilfe. Gerade hier war es möglich in einem Wohlfahrtsverband ein innovatives Projekt umzusetzen. Dabei wirkte sich der Einfluss der Kommune eher fördernd auf das Projekt aus.

„.Grundsātzlich, cinmal so zur Erklẫrung. Es ist in [Name der Stadt] so, dass die Zusammenarbeit mit der Stadt [ ...] auf Kooperation ausgelegt ist. Es gibt das sogenannte stadtweite Netzwerk. Da hat die Koordination die Stadt [...]. In diesern stadtweiten Netzwerk sind alle Anbieter - sag ich mal - alle Träger. Sind es nicht nur die Wohlfahrtsverbände...Nein es ist auch der Seniorenbeirat vertreten, die versehiedenen Träger kommen da zusammen und besprechen dies oder jenes was da in der Seniorenlandschaft gerade los ist, was es zu koordinieren gibt, wo es irgendetwas zusammen zu gestalten gibt. Das ist hier der Boden, der hier gelegt wurde, um 2003 rum. Das transportiert sich in die Stadtbezirke. [...] Da gibt es immer einen stådtischen Sozialarbeiter der koordiniert, das stadtbezirkliche Netzwerk und da kommen dann alle zusammen. Da trifft dann auch die Kollegin von der [Namen des Wohlfahrtsverbandes] Scniorenarbeit viclleicht den F. vom Pflegedienst oder jemand ganz andercs von [Name cines Wohlfahrtsverbandes], oder der Kirche. Also das ist so das Grundgerüst hier und das ist schon mal viel wert. Das ist schon einmal eine gute Basis, um überhaupt voneinander zu wissen und zu gucken, was machen dic einen, was machen die anderen." (Interviewauszug Care I)

Anhand der beiden Fallbeispiele im Handlungsfeld kultursensible Altenhilfe zeigt sich wie stark der Einfluss von Trägern und Kommunen abhängig ist von einzelnen Persönlichkeiten und deren Engagement.

\section{Die Bedeutung von Social Entrepreneurship in Deutschland}

Die dargestellten empirischen Befunde zeigen, dass das Phänomen des aus dem angelsächsischen Raum importierten „Social Entrepreneurship "in einem durch etablierte und vernetzte Strukturen gekennzeichneten Wohlfahrtsstaat eine weniger ausgeprägte Rolle spielt. In Deutschland wird die Erbringung sozialer Dienstleistungen traditionell von intermediären Akteuren zwischen Staat, Markt und 
Gesellschaft organisiert, so dass das Spielfeld bereits weitgehend besetzt ist. Es stellt sich die Frage, ob Social Entrepreneurship im engeren Sinne, als marktförmige, unternehmerische Lösung sozialer Probleme, über den Einzelfall hinaus in der Lage ist, effizienter und effektiver zu agieren als Staat und Verbände, die ihrerseits ihre Legitimation aus der Bearbeitung von durch Marktversagen verursachten Problemen ziehen.

Es hat sich gezeigt, dass es sich bei den (vermeintlichen) Social Entrepreneurs oftmals weder um inhaltlich besonders innovative noch um eine originär neue Form der Leistungserstellung handelt, die gegenüber den Angeboten etablierter Träger über spezifische Vorteile verfügen, sondern dass etablierte Akteure ähnlich agieren können und ggf. neue Ideen und Strukturen innerhalb etablierter Organisationen entwickeln (Intrapreneurship). Auch in den etablierten Organisationen, den Wohlfahrtsverbänden, wurden im Zuge der Einführung neuer Steuerungsmodelle bereits seit mehr als 15 Jahren betriebswirtschaftliche Instrumente der Unternehmensführung und -steuerung eingeführt (vgl. u.a. Grohs/ Bogumil 2011; Dahme/Kühnlein/Wohlfahrt 2005; Liebig 2005; die Beiträge in Evers/Heinze 2008; Schneiders 2010: 57f). Auch wenn dieser Prozess von Teilen der Akteure der Sozialen Arbeit als „Ökonomisierung“ bzw. „Verbetriebswirtschaftlichung" diskreditiert wird (vgl. für eine differenzierte Auseinandersetzung Möring-Hesse 2008; für eine emotionale Seithe 2011), zeigt doch der rasante Anstieg der Zahl der Studiengänge des Sozialmanagements und der Sozialwirtschaft (Boeßenecker/Markert 2007), dass die Notwendigkeit einer stärkeren Wirkungsorientierung von einem Großteil der etablierten Akteure erkannt worden ist (vgl. die Beiträge in Epler/Miethe/Schneider 2011).

Die von uns identifizierten Projekte entsprechen nur in geringer Zahl dem in der Social Entrepreneurship-Literatur gefeierten philanthropischen Unternehmertypus, der mit etablierten Strukturen bricht, Neues auf die Beine stellt und durch die Diffusion seines Ansatzes den deutschen Sozialstaat transformiert. Vielmehr handelt es sich in der Regel um Projekte, die sich aus bestehenden Strukturen heraus entwickeln (Intrapreneurship) und von konkreten Problemlagen angestoßen werden. Innovation entsteht vielmehr insbesondere dort, wo etablierte Akteure zusammenarbeiten und gemeinsame, oft hybride Lösungen finden. Dieses Ergebnis weist zumindest in den von uns untersuchten Handlungsfeldern auf eine nur beschränkte empirische Relevanz des Typus „Social Entrepreneur“ im engeren Sinne hin. Zwar bestätigen die Ergebnisse, dass im bereits stärker ökonomisierten Handlungsfeld der kultursensiblen Altenhilfe, verstärkt neue Akteure neben die Etablierten treten, jedoch wird in der Empirie ebenfalls deutlich, dass sich diese wiederum durch eine starke Vernetzung mit etablierten Akteuren aus- 
zeichnen. Die Tatsache, dass wir in beiden Feldern nur eine relativ geringe Zahl von Social Entrepreneurs identifizieren konnten, mag zum Teil auf das methodische Design zurückzuführen sein.

Bezüglich unserer These, dass sich korporatistische Strukturen bzw. rechtliche Rahmenbedingungen hemmend auswirken, ergibt sich ein uneinheitliches Bild: Während sich die Befragten der quantitativen Analyse relativ zufrieden mit den Rahmenbedingungen zeigten, äußerten die Akteure in den Fallstudien zum Teil erhebliche Kritik. Kontrastiert man unsere empirischen Befunde mit der diskursiven Prominenz von SE, ist fraglich, ob sich ein Paradigmenwechsel vollziehen wird. Es ist unbestritten, dass das Konzept des Social Entrepreneurship aufgrund seines vermeintlichen visionären Charakters eine hohe Attraktivität aufweist - insbesondere in Kreisen außerhalb des engeren Policy-Netzwerks der Sozialpolitik. Hier eröffnet sich (scheinbar) ein Ausweg aus dem Dilemma der wachsenden sozialen Aufgaben bei stagnierenden öffentlichen Finanzen. Zudem wird ein qualitativer Mehrwert durch das persönliche Engagement der „Unternehmer" im Bereich der sozialen Dienstleistungen erwartet. Die Ausführungen zu den Begrifflichkeiten sowie die Darstellung der empirischen Realitäten im deutschen sozialen Dienstleistungssektor haben jedoch gezeigt, dass die Übertragung eines Modells aus dem angelsächsischen Raum auf die deutsche „Wohlfahrtsstaatswirklichkeit" nur begrenzt möglich ist. Vielmehr müssen zunächst Begrifflichkeiten und Konzepte an das jeweilige wohlfahrtsstaatliche Regime angepasst werden und die jeweiligen institutionellen Kontexte einbezogen werden. Im Rahmen unserer quantitativen und qualitativen Untersuchungen ist zudem deutlich geworden, dass Social Entrepreneurship als Selbstbeschreibung der Akteure im Sozialbereich bislang kaum auftritt, so dass wir dieses Kriterium zu Recht nicht zur Fallauswahl genutzt haben.

Welchen Stellenwert haben nun die von uns identifizierten Beispiele von Social Entrepreneurship? Tatsächliche Konkurrenz zwischen etablierten Formen der Wohlfahrtsproduktion und den neuen Initiativen des „Social Entrepreneurship“ ist bislang - auch angesichts des noch bescheidenen Ausmaßes und unterschiedlicher Zielgruppen von SE - noch nicht erkennbar. Die Projekte bewegen sich einerseits in Nischen und „stemmen“ kein allzu großes Risiko. Damit wird ein mögliches Scheitern in seinen Auswirkungen begrenzt. Etablierte Träger werden so kaum unter Druck gesetzt. Allerdings entwickeln die Modelle eine ,andere“ Form sozialen Handelns, die von Etablierten durchaus gesehen wird - und nicht selten in das eigene Handlungsrepertoire integriert wird. SEs entwickeln in diesem Kontext $z$. T. neue Ansätze, indem sie bislang engagementferne Gruppen gezielt ansprechen (z. B. Schüler mit Migrationshintergrund im IBFS - Chancenwerk) oder bewusst auf ,hippe“ Organisationsformen setzen (z. B. „Rock your life“). Insofern 
bieten neue innovative Projekte, das Potential neues Engagement zu generieren. Nicht zuletzt dürfte der überwiegende Teil der sich als SEs bezeichnenden Projekte aus privatem Engagement entstanden sein, so dass sich hier - nicht unvergleichbar mit der Institutionalisierung der Selbsthilfebewegung der 1970er und 1980er Jahre - eine Institutionalisierung von Engagement vollzieht.

Ein Wettbewerb um Engagierte und Arbeitskräfte ist bislang nicht erkennbar. Allerdings könnte die gegenwärtige Aufmerksamkeit für SEs, die nicht zuletzt durch nicht unerheblichen Aufwand von Mittlerorganisationen geschaffen wird, zu zwei nicht intendierten, aber problematischen Effekten führen. Angesichts der medialen Präsenz ist zu befürchten, dass Initiativen „altmodischen“ Zuschnitts in der neuen Aufmerksamkeitsökonomie einen geringeren Anteil des Kuchens am Aufkommen von Spenden und Fördermitteln abbekommen könnten, bzw. gezwungen werden, mit ähnlichen medialem Aufwand um diese zu werben - was zu einer Verschiebung ihres Arbeitsfokus weg von der eigentlichen Problembearbeitung hin zu Vermarktung führt. Dies schließt die Gefahr des „Rosinenpickens" hinsichtlich der Zielgruppen ein, so dass die Aufmerksamkeit auf Gruppenverschoben wird, bei denen leichter medial vermittelbare Erfolge erzielt werden können. Zum zweiten etablieren sich gegenwärtig neue SE nahe Formen von „Rating-Agenturen“ für Sozialprojekte, die potentiellen Mäzenen Handreichungen für „Social Investments“ bereitstellen (z. T. mit erheblichen Kosten). Die sich etablierenden Systeme der Wirkungsmessung laufen relativ unverbunden zur lange anhaltenden Debatte um Wirkungsorientierung in der sozialen Arbeit (vgl. für einen Überblick Otto 2007)und dem öffentlichen Sektor, so dass die Gefahr besteht, dass eine sehr spezifische, weitgehend nicht öffentlich gesetzte Perspektive über die Verteilung von Mitteln bestimmt.

Dringlicher als die Beachtung dieser potentiellen Probleme ist allerdings das Erfordernis der Koordination zwischen Angeboten bzw. Akeuren. Kooperation, Vernetzung und mehr Wettbewerb und Management sind die Schlüsselfragen im Bereich sozialer Dienstleistungen. Die bisher nebeneinander stehenden Einrichtungen müssen ,neu“ vernetzt werden, so dass Reibungsverluste verhindert und Ressourcen gebündelt werden in Richtung des Aufbaus einer lokalen sozialen Infrastruktur. Die neuen Akteure im Sozialsektor (SEs) sollten hier beachtet, aber ihre Relevanz(nach den bisher vorliegenden Befunden) nicht überschätzt werden. SEs können als Innovationsinkubator fungieren, aber ihre Ausstrahlungskraft ist dann am größten, wenn sie in Kooperation mit bzw. innerhalb der etablierten Strukturen (Intrapreneurship) agieren. Insofern bewirken SEs in etablierten Wohlfahrtsstaaten nicht „Change“ im Sinne weitreichenden Strukturwandels, sondern das Setzen kleiner feiner Unterschiede. 


\section{Literaturverzeichnis}

Arbcitskreis „Charta für cinc kultursensible Altenpflcgc“( Hrsg) (2002): Handreichung für cinc kultursensible Altenpflege, Köln: o. V.

Ashoka (2010): Jahresbericht 2009, Berlin: o.V., download unter http://germany.ashoka.org/sites/ germany.ashoka.org/files/Ashoka_JB_2009\%20final\%20Web.pdf. letzter Zugriff 02.11.2010.

Billis, David (Hg.) (2010): Hybrid Organizations and the Third Sector: Challenges for Practice, Theory and Policy. Basingstoke: Palgrave Macmillan.

Bode, Ingo (2004): Disorganisierter Wohlfahrtskapitalismus Dic Reorganisation des Sozialscktors in Deutschland, Frankreich und Großbritannien, Wiesbaden: Verlag für Sozialwissenschaften 2004

Bode, Ingo/ Evers, Adalbert (2004): From institutional fixation to entrepreneurial mobility'? The German third sector and its contemporary challenges. In: Evers, Adalbert/ Laville,Jean-Louis ( $\mathrm{Hg}$.): The third sector in Europe. Cheltenham: Elgar, S. 101-121.

Boeßenecker, Karl-Heinz/Markert, Andreas (2007): Sozialmanagement studieren. Studienangebotc im Bereich Sozialmanagement und Sozialwirtschaftund Analysen veränderter Rahmenbedingungen, Dūsseldorf: HBS (Arbcitspapier 141).

Borins, Sandford (2001): The Challenge of Innovating in Government: PricewaterhouseCoopers Endowment for the Business of Government.

Bornstein, David (2007): How to change the world. Social entrepreneurs and the power of new ideas. Oxford: Oxford University Press.

Bornstein, David/ Davis, Susan (2010): Social entreprencurship. What everyone necds to know. Oxford, New York: Oxford University Press.

Brandsen, Taco; Dekker, Paul; Evers, Adalbert (2010): Civicness in the governance and delivery of social services. Baden-Baden: Nomos.

Dahme/Heinz-Jürgen/Kühnlein, Gertrud/Wohlfahrt, Norbert (2005): Zwischen Wettbewerb und Subsidiarität.Wohlfahrtsverbände unterwegs in die Sozialwirtschaft, Berlin: edition sigma.

Decs, J. Gregory/Anderson, Bcth Battlc (2006): Framing a Theory of Social Entreprencurship: building on Two Schools of Practice and Thought. In: Mosher-Williams, R., Association for Research on Nonprofit Organizations und Voluntary Action (Hg.): Research on social entrepreneurship: understanding and contributing to an emerging field: Aspen Institute (ARNOVA oecasional paper series), S. 39-66.

Defourny, Jacques/ Nyssens, Marthe (2010): Conceptions of Social Enterprise and Social Entrepreneurship in Europe and the United States: Convergenecs and Divergences. In: Journal of Social Entrepreneurship 1 (1), S. 32-53.

Edwards, Michael (2010): Small Change. Why BusinessWon't Save the World. C San Francisco: Berrett-Koehler Publishers.

Elkington, John/Hartigan, Pamela (2008): The power of unreasonable people. How social entrepreneurs create markets that change the world. Boston: Harvard Business School Press.

Eppler, Natalie/Miethe, Ingried/Schneider, Armin (2011): Qualitative und quantitative Wirkungsforschung: Ansätze, Beispiele, Perspektiven. Opladen: Verlag Barbara Budrich

Evers, Adalbert (1995): Part of the welfare mix: The third sector as an intermediate area. In: Voluntas 6 (2), S. 159-182.

Evers, Adalbert/ Ewert, Benjamin (2010): Hybridc Organisationen im Bereich sozialer Dienste Ein Konzept, sein Hintergrund und seine Implikationen. In: Klatetzki, Thomas (Hg.): Soziale personenbezogene Dienstleistungsorganisationen. Soziologische Perspektiven. Wiesbaden: VS Verlag, S. 103-128. 
Evers, Adalbert/Heinze, Rolf G. (Hrsg.) (2008): Sozialpolitik. Ökonomisierung und Entgrenzung, Wiesbaden: VS Verlag.

Evers, Adalbert/Heinze, Rolf G./Olk, Thomas (Hg.) (2011): Handbuch Soziale Dienste, Wiesbaden

Gogolin, Ingrid/ Neumann, Ursula/ Roth, Hans-Joachim (2003): Förderung von Kindern und Jugendlichen mit Migrationshintergrund. Gutachten. Bonn: BLK (Materialien zur Bildungsplanung und zur Forschungsförderung, 107).

Grohs, Stephan (2010): Modernisierung kommunaler Sozialpolitik. Wiesbaden: VS Verlag.

Grohs, Stephan/Bogumil, Jörg (2011): Management sozialer Dienste. In:Evers, Adalbert/Heinze, Rolf G./Olk, Thomas (Hg.): Handbuch Soziale Dienste, Wiesbaden

Hackenberg, Helga/ Empter, Stefan (Hg.) (2011): Social Entrepreneurship im deutschen Wohlfahrtsstaat - Hybride Organisationen zwischen Markt, Staat und Gemeinschaft. Wiesbaden: VS Verlag.

Heinze, Rolf G./ Naegele, Gerhard (2010): Integration und Vernetzung - Soziale Innovationen im Bereich sozialer Dienste. In: Howaldt, Jürgen/ Jacobsen, Heike (Hg.): Soziale Innovation. Auf dem Weg zu einem postindustriellen Innovationsparadigma. Wiesbaden: VS Verlag, S. 297-313.

Heinze, Rolf G./ Grohs, Stephan/ Schneiders, Katrin (2011): Social Entrepreneurship im deutschen Wohlfahrtsstaat - Hybride Organisationen zwischen Markt, Staat und Gemeinschaft. In: Hackenberg, Helga/ Empter, SteFan (Hg.): Social Entrepreneurship im deutschen Wohlfahrtsstaat - Hybride Organisationen zwischen Markt, Staat und Gemeinschaft. Wiesbaden: VS Verlag, S. 86-102.

Jähnke, Petra/ Christmann, Gabricla B./ Balgar, Karsten (Hg.) (2011): Social Entrepreneurship. Perspektiven für die Raumentwicklung Wiesbaden: VS Verlag.

Jansen, Stephan A./Richter, Saskia/Hahnke, Elisabeth/Achleitner, Ann-Kristin/Spiess-Knafl, Wolfgang/Volk, Sarah/Then, Volker/Mildenberger, Georg/Scheuerle, Thomas/Schmitz, Blörn (2010): Defining Social Entrepreneurship (Eine Definition von Social Entrepreneurship). Zeppelin Universität. Friedrichshafen, Heidelberg, München (Working Paper).

Kerlin, Janelle A. (2006): Social Enterprise in the United States and Europe: Understanding and Learning from the Differences. In: Voluntas 17 (3), S. 246-262.

Kerlin, Janelle A. (2012): Defining Social EnterpriseAcross Different Contexts: A Conceptual FrameworkBased on InstitutionalFactors. In: Nonprofit and Voluntary Sector Quarterly 20 (10) 1-12. Kirzner, Israel M. (1999): Creativity and/or Alertness: A Reconsideration of the Schumpeterian Entrepreneur. In: The Review of Austrian Economics 11 (1/2), S. 5-17.

Kirzner. Israel M (1999): Creativity and/or Alertness: A Reconsideration of the Schumpeterian Entrepreneur, In: The Review of Austrian Economics 11 (1-2), 5-17.

Liebig, Reinhard (2005): Wohlfahrtsverbände im Őkonomisierungsdilemma. Freiburg: Lambertus.

Möring-Hesse, Matthias (2008): Verbetriebswirtschaftlichung und Verstaatlichung. Die Entwicklung der sozialen Dienste und der Freien Wohlfahrtspflege, in: Zeitschrift für Sozialreform H. 2 (54.Jg.), S. 141-160.

Mulgan, Geoff/Albury, David (2003): Innovation in the Public Sector, Working Paper Version 1.9, October, Strategy Unit, UK Cabinet Office.

Nicholls, Alex (Hg.) (2006): Social Entrepreneurship. New Models of Sustainable Social Change. Oxford: Oxford University Press.

Otto, Hans-Uwe (2007): Zum aktuellen Diskurs um Ergebnisse und Wirkungen im Feld der Sozialpädagogik und Sozialarbeit - Literaturvergleich nationaler und internationaler Diskussion. Expertise im Auftrag der Arbeitsgemeinschaft für Kinder- und Jugendhilfe-AGJ. Berlin. 
Perrini, Francesco/Vurro, Clodia (2006): Social entrepreneurship: Innovation and social change across theory and practice. In: Mair, Johanna/ Robinson, Jeffrey/ Hockerts, Kai (Hg.): Social entrepreneurship. Basingstoke. Hampshire: Palgrave Macmillan.

Salamon, Lester M./ Anheier, Helmut (1996): The emerging nonprofit sector. An overview. Manchester: Manchester Univ. Press.

Schneiders, Katrin (2010): Vom Altenheim zum Seniorenservice. Institutioneller Wandel und Akteurkonstellationen im sozialen Dienstleistungssektor-Baden-Baden: Nomos.

Seithe, Mechthild (2011): Schwarzbuch Soziale Arbeit. 2. Aufl., Wiesbaden: VS-Verlag

Teasdale, Simon (2012): What's in a Name? Making Sense of Social Enterprise Discourses. In: Public Policy and Administration 27 (2), S. 99-119.

Zimmer, Annette (2007): Vereine - Zivilgesellschaft konkret. Wiesbaden: VS Verlag. 